\title{
GLOBAL EXISTENCE AND DECAY OF SOLUTIONS OF A COUPLED SYSTEM OF BBM-BURGERS EQUATIONS
}

\author{
Jardel MORAIS PEREIRA*
}

\begin{abstract}
The global well-posedness of the initial-value problem associated to the coupled system of BBM-Burgers equations

$$
\begin{aligned}
& u_{t}-u_{x x t}-a_{3} v_{x x t}+u^{p} u_{x}+a_{1} v^{p} v_{x}+a_{2}\left(u^{p} v\right)_{x}-\varepsilon_{1} u_{x x}=0 \\
& b_{1} v_{t}-v_{x x t}-b_{2} a_{3} u_{x x t}+v^{p} v_{x}+b_{2} a_{2} u^{p} u_{x}+b_{2} a_{1}\left(u v^{p}\right)_{x} \\
& -\varepsilon_{2} v_{x x}=0
\end{aligned}
$$

in the classical Sobolev spaces $H^{s}(\mathbb{R}) \times H^{s}(\mathbb{R})$ for $s \geq 2$ is studied. Furthermore we find decay estimates of the solutions of $\left(^{*}\right)$ in the norm $L^{q}(\mathbb{R}) \times L^{q}(\mathbb{R}), 2 \leq q \leq \infty$ for general initial data provided that $a_{3}^{2} b_{2}<1$ and $p \geq 3$. Model $(*)$ is motivated by a work due to Gear and Grimshaw [10] who considered strong interaction of weakly nonlinear long waves governed by a coupled system of $\mathrm{KdV}$ equations.
\end{abstract}

\section{Introduction}

We consider the initial value problem for a coupled system of BBMBurgers equations

$$
\begin{aligned}
& u_{t}-u_{x x t}-a_{3} v_{x x t}+u^{p} u_{x}+a_{1} v^{p} v_{x}+a_{2}\left(u^{p} v\right)_{x}-\varepsilon_{1} u_{x x}=0 \\
& b_{1} v_{t}-v_{x x t}-b_{2} a_{3} u_{x x t}+v^{p} v_{x}+b_{2} a_{2} u^{p} u_{x}+b_{2} a_{1}\left(u v^{p}\right)_{x}-\varepsilon_{2} v_{x x}=0
\end{aligned}
$$

with initial conditions

$$
u(x, 0)=\varphi_{1}(x), \quad v(x, 0)=\varphi_{2}(x)
$$

*Partially supported by FINEP (Brasil).

1991 Mathematics Subject Classification: 35Q53, 35B40.

Servicio de Publicaciones. Universidad Complutense. Madrid, 2000 
where $u=u(x, t), v=v(x, t)$ are real functions of the real variables $-\infty<x<\infty, t \geq 0$. All coefficients in (1.1) are real constants with $b_{1}$, $b_{2}, \varepsilon_{1}, \varepsilon_{2}$ positive and the power $p$ is an integer bigger than or equal to one.

To motivate our study let us mention some works related with (1.1). The following coupled system

$$
\begin{aligned}
& u_{t}+u_{x x x}+a_{3} v_{x x x}+u^{p} u_{x}+a_{1} v^{p} v_{x}+a_{2}\left(u^{p} v\right)_{x}-\varepsilon u_{x x}=0 \\
& b_{1} v_{t}+r v_{x}+v_{x x x}+b_{2} a_{3} u_{x x x}+v^{p} v_{x}+b_{2} a_{2} u^{p} u_{x}+b_{2} a_{1}\left(u v^{p}\right)_{x} \\
& -\varepsilon v_{x x}=0
\end{aligned}
$$

was originally derived in the case $p=1$ and $\varepsilon=0$ by J. A. Gear and R. Grimshaw in 1984 [10] as a model to describe the strong interaction of weakly nonlinear, long waves. The Cauchy problem for (1.3) with $p=1$ and $\varepsilon=0$ was studied by Bona, Ponce, Saut and Tom [8], and by Marshall Ash, Cohen and Wang [12].

The asymptotic behaviour in time of solutions of (1.3) was studied by E. Bisognin, V. Bisognin and G. Perla Menzala in [3]. In particular, for $\varepsilon>0$, they obtained the following decay estimate

$$
\|u(\cdot, t)\|_{L^{q}}+\|(v \cdot, t)\|_{L^{q}} \leq C t^{\frac{1-q}{2 q}}, \quad \text { as } t \rightarrow \infty
$$

for any $2 \leq q \leq \infty$, provided that the initial data are sufficiently small, $p>4$ and $a_{3}^{2} b_{2}<1$.

Concerning model (1.1), V. Bisognin [5] proved global existence and the asymptotic behaviour of solutions when $\varepsilon_{1}=\varepsilon_{2}=0$ and $b_{1}=1$, where, under suitable smallness assumptions on the initial data and $p>4$ the following $L^{\infty}$ decay estimates are obtained

$$
\|u(\cdot, t)\|_{L^{\infty}} \leq C(1+t)^{-\frac{1}{3}}, \quad\|v(\cdot, t)\|_{L^{\infty}} \leq C(1+t)^{-\frac{1}{3}} \text { as } t \rightarrow \infty .
$$

Observe that if we consider $a_{1}=a_{2}=a_{3}=0$ in (1.1) and (1.3) we obtain, respectively, BBM-Burgers and KdV-Burgers type equations, which are well known model equations for long waves in nonlinear dispersive media where dissipative effects are considered. The existence and decay of solutions for these (and related) equations has been intensively studied by several authors in recent years (see [1], [2], [6], [7] for example and the references therein). 
In this paper we study the global well-posedness and decay of solutions of the initial-value problem associated to the system (1.1) in the case $\varepsilon_{1}>0, \varepsilon_{2}>0$. We rewrite (1.1)-(1.2) as follows

$$
\begin{aligned}
& b_{1} U_{t}-A U_{x x t}+F(U)_{x}=B U_{x x} \\
& U(x, 0)=\varphi(x)
\end{aligned}
$$

where

$$
\begin{array}{cc}
U=\left(\begin{array}{l}
u \\
v
\end{array}\right), & \varphi=\left(\begin{array}{l}
\varphi_{1} \\
\varphi_{2}
\end{array}\right) \\
A=\left[\begin{array}{cc}
b_{1} & b_{1} a_{3} \\
b_{2} a_{3} & 1
\end{array}\right], \quad B=\left[\begin{array}{cc}
\varepsilon_{1} b_{1} & 0 \\
0 & \varepsilon_{2}
\end{array}\right]
\end{array}
$$

and the components of $F(U)$ are given by

$$
\begin{aligned}
& F_{1}(U)=\frac{b_{1}}{p+1} u^{p+1}+b_{1} a_{2} u^{p} v+\frac{b_{1} a_{1}}{p+1} v^{p+1} \\
& F_{2}(U)=\frac{b_{2} a_{2}}{p+1} u^{p+1}+b_{2} a_{1} u v^{p}+\frac{1}{p+1} v^{p+1}
\end{aligned}
$$

A local solution of (1.4)-(1.5) with initial data in $H^{s}(\mathbb{R}) \times H^{s}(\mathbb{R})$ for $s \geq 2$ and $a_{3}^{2} b_{2}<1$ can easily be obtained by inverting the operator $M=b_{1} I-A \frac{\partial^{2}}{\partial x^{2}}$ in (1.4) and applying Banach Fixed Point Theorem to the integral equation associated with (1.4)-(1.5). Here $I$ denotes the $2 \times 2$ identity matrix. To extend the local solution to a global solution we use the regularization property of the operator $M^{-1} \circ \frac{\partial}{\partial x}$ together with an $H^{1} \times H^{1}$ a priori estimate (energy estimate). See sections 2 and 3 .

Due to the dissipative terms $\varepsilon_{1} u_{x x}, \varepsilon_{2} v_{x x}$ the energy associated to the linear system, that is (1.1) without the nonlinear terms, decays to zero as $t \rightarrow \infty$, so that we could expect the same behaviour for the solutions of (1.1)-(1.2) (or equivalently (1.4)-(1.5)). In section 4 we prove that this in fact happens provided $p \geq 3$. More precisely, if $\varphi \in H^{s}(\mathbb{R}) \times H^{s}(\mathbb{R})$ with $s \geq 2, a_{3}^{2} b_{2}<1$ and $p \geq 3$, then using our previous analysis on the linear part studied in section 2 we obtain the following decay estimates for the solution of (1.4)-(1.5):

(i) $\|U(\cdot, t)\|_{L^{\infty} \times L^{\infty}} \leq C(1+t)^{-\frac{1}{4}}\|\varphi\|_{H^{1} \times H^{1}}$

(ii) $\|U(\cdot, t)\|_{L^{q} \times L^{q}} \leq C(1+t)^{-\frac{1}{2}\left(1-\frac{1}{q}\right)}\left(\|\varphi\|_{H^{1} \times H^{1}}+\|\varphi\|_{L^{1} \times L^{1}}\right)$

for all $2 \leq q \leq \infty$, where in (ii) we also assume that $\varphi \in L^{1}(\mathbb{R}) \times L^{1}(\mathbb{R})$. 
We remark that the above results are proved without any restriction on smallness of the initial data. However the technique used in this paper to obtain (i)-(ii) does not apply to the case of $1 \leq p<3$. Recently, for the case $p=1$, the decay in time of space-periodic solutions of a generalization of system (1.1) was studied by E. Bisognin, V. Bisognin and G. Perla Menzala in [4].

Some notations used in this paper are as follows. In general, for $X$ and $Y$ Banach spaces, the norm of $f$ in $X$ will be denoted by $\|f\|_{X}$ and in the product $X \times Y$ we always consider the norm $\|(f, g)\|_{X \times Y}^{2}=$ $\|f\|_{X}^{2}+\|g\|_{Y}^{2}$. For $1 \leq p \leq \infty$ we denote by $L^{p}=L^{p}(\mathbb{R})$ the Banach space of real-valued functions defined on $\mathbb{R}$ whose $p$-th power is Lebesgue integrable (essentially bounded in the case $p=\infty$ ) with the usual norm. In the particular case $p=2$ we will denote by $(,)_{L^{2}}$ the inner product of $L^{2}$. We denote by $\hat{f}$ the Fourier transform of a function $f$. If $f \in$ $L^{2}(\mathbb{R})$ then $\hat{f} \in L^{2}(\mathbb{R})$ and the Fourier transform can be extended to an isomorphism from $L^{2}(\mathbb{R})$ onto itself. For any real number $s, H^{s}=H^{s}(\mathbb{R})$ denotes the Sobolev space of order $s$. We will denote by $C$ a generic constant which value can be vary from line to line.

\section{Linear estimates}

We consider the linear system

$$
\begin{aligned}
& u_{t}-u_{x x t}-a_{3} v_{x x t}-\varepsilon_{1} u_{x x}=0 \\
& b_{1} v_{t}-b_{2} a_{3} u_{x x t}-v_{x x t}-\varepsilon_{2} v_{x x}=0
\end{aligned}
$$

with initial conditions

$$
u(x, 0)=\varphi_{1}(x), \quad v(x, 0)=\varphi_{2}(x) .
$$

Our aim in this section is to study some decay estimates of the solutions of (2.1)-(2.2) and prove some facts which will be used in the next sections.

With the notation of the introduction we rewrite (2.1)-(2.2) as

$$
b_{1} U_{t}-A U_{x x t}=B U_{x x}, \quad U(x, 0)=\varphi(x)
$$

where $A$ and $B$ are as in (1.6), and $\varphi=\left(\begin{array}{l}\varphi_{1} \\ \varphi_{2}\end{array}\right)$. 
Let us consider equation (2.3) in the Sobolev space $H^{s} \times H^{s}$ for $s \geq 2$. Recall that $M$ denotes the operator $M=b_{1} I-A \frac{\partial^{2}}{\partial x^{2}}$.

Lemma 2.1. Let $a_{3}^{2} b_{2}<1$ and $s \geq 2$. Then the operator $M: H^{s} \times H^{s} \rightarrow$ $H^{s-2} \times H^{s-2}$ is invertible and its inverse $M^{-1}$ is given by $M^{-1} g=K * g$, where $K=\left(k_{j \ell}\right)$, with $k_{j \ell} \in L^{1} \cap L^{\infty}, j, \ell=1,2$, and * denotes spatial convolution.

Proof. The hypothesis $a_{3}^{2} b_{2}<1$ implies that the matrix $A$ has two positive eigenvalues $\lambda_{1}, \lambda_{2}$. Let $P$ a nonsingular matrix such that $P^{-1} A P$ is equal to $D=\left[\begin{array}{cc}\lambda_{1} & 0 \\ 0 & \lambda_{2}\end{array}\right]$. Given $g \in H^{s-2} \times H^{s-2}$ consider the equation $b_{1} V-D V_{x x}=P^{-1} g$. Using the Fourier transform we see that this equation has a unique solution $V \in H^{s} \times H^{s}$ for $s \geq 2$, which is given by

$V=\left[\begin{array}{lr}L_{1} & 0 \\ 0 & L_{2}\end{array}\right] * P^{-1} g, \quad$ where $\quad L_{j}(x)=\frac{1}{2 b_{1}} \sqrt{\frac{b_{1}}{\lambda_{j}}} \exp \left(-|x| \sqrt{\frac{b_{1}}{\lambda_{j}}}\right)$,

$j=1,2$. Consequently, $U=P V$ is the unique solution of $M U=g$. This shows that $M$ is invertible. To find $M^{-1}$, let $P=\left(p_{j \ell}\right), P^{-1}=\left(q_{j \ell}\right)$, $U=\left(\begin{array}{l}u_{1} \\ u_{2}\end{array}\right)$ and $g=\left(\begin{array}{l}g_{1} \\ g_{2}\end{array}\right)$. From $U=P V$ we obtain

$$
\begin{aligned}
u_{j} & =p_{j 1} L_{1} *\left(q_{11} g_{1}+q_{12} g_{2}\right)+p_{j 2} L_{2} *\left(q_{21} g_{1}+q_{22} g_{2}\right) \\
& =\left(p_{j 1} q_{11} L_{1}+p_{j 2} q_{21} L_{2}\right) * g_{1}+\left(p_{j 1} q_{12} L_{1}+p_{j 2} q_{22} L_{2}\right) * g_{2},
\end{aligned}
$$

$j=1,2$. This shows that $M^{-1} g=K * g$, with $K=\left(k_{j \ell}\right)$, where $k_{j \ell}=p_{j 1} q_{1 \ell} L_{1}+p_{j 2} q_{2 \ell} L_{2}$ are all in $L^{1} \cap L^{\infty}$.

Corollary 2.2. For any $g \in H^{s} \times H^{s}$ with $s \geq 0$, there exists a positive constant $C_{0}$ such that $\left\|M^{-1} g\right\|_{H^{s+1} \times H^{s+1}} \leq C_{0}\|g\|_{H^{s-1} \times H^{s-1}}$.

Proof. The components of the vector $M^{-1} g$ are

$$
\begin{aligned}
& k_{j 1} * g_{1}+k_{j 2} * g_{2}=p_{j 1} q_{11} L_{1} * g_{1}+p_{j 2} q_{21} L_{2} * g_{1} \\
& +p_{j 1} q_{12} L_{1} * g_{2}+p_{j 2} q_{22} L_{2} * g_{2}, \quad j=1,2
\end{aligned}
$$

where $g=\left(\begin{array}{l}g_{1} \\ g_{2}\end{array}\right)$. Thus,

$$
\left\|M^{-1} g\right\|_{H^{s+1} \times H^{s+1}} \leq \sum_{j=1}^{2}\left\|k_{j 1} * g_{1}+k_{j 2} * g_{2}\right\|_{H^{s+1}}
$$




$$
\begin{aligned}
& =\sum_{j=1}^{2} \| p_{j 1} q_{11} L_{1} * g_{1}+p_{j 2} q_{21} L_{2} * g_{1}+p_{j 1} q_{12} L_{1} * g_{2} \\
& +p_{j 2} q_{22} L_{2} * g_{2}\left\|_{H^{s+1}} \leq C_{0}\right\| g \|_{H^{s-1} \times H^{s-1}}
\end{aligned}
$$

where

$$
C_{0}=\max \left\{\frac{1}{\min \left(b_{1}, \lambda_{1}\right)}, \frac{1}{\min \left(b_{1}, \lambda_{2}\right)}\right\}\|P\|\left\|P^{-1}\right\|,
$$

with $\|P\|=\sum_{j, \ell=1}^{2} \mid p_{j \ell \mid}$ as

Using Lemma 2.1 we can write (2.3) in the space $H^{s} \times H^{s}$ with $s \geq 2$

$$
U_{t}=\mathcal{L} U, \quad U(\cdot, 0)=\varphi,
$$

where $\mathcal{L}=M^{-1} B \frac{\partial^{2}}{\partial x^{2}}$. The initial value problem (2.4) has a unique solution $U \in C^{1}\left([0, \infty) ; H^{s} \times H^{s}\right)$, which is given by $U(\cdot, t)=E(t) \varphi$, where $E(t)$ denotes the semigroup of linear operators on $H^{s} \times H^{s}$ generated by $\mathcal{L}$. Therefore, the components $u, v$ of $U$ are the unique functions in $C^{1}\left([0, \infty] ; H^{s}\right)$ satisfying $(2.1)-(2.2)$.

Next we will find explicit formulas for $u$ and $v$ which will allow us to derive some decay estimates.

Taking the Fourier transform of (2.1) and (2.2) we obtain

$$
\widehat{U}_{t}(z, t)+A(z) \widehat{U}(z, t)=0, \quad \widehat{U}(z, 0)=\hat{\varphi}(z)
$$

where $\widehat{U}=\left(\begin{array}{l}\hat{u} \\ \hat{v}\end{array}\right), \quad \hat{\varphi}=\left(\begin{array}{c}\hat{\varphi}_{1} \\ \hat{\varphi}_{2}\end{array}\right)$ and

$$
A(z)=\frac{z^{2}}{\sigma(z)}\left[\begin{array}{cc}
\left(b_{1}+z^{2}\right) \varepsilon_{1} & -a_{3} z^{2} \\
-b_{2} a_{3} \varepsilon_{1} z^{2} & \left(1+z^{2}\right) \varepsilon_{2}
\end{array}\right]
$$

with $\sigma(z)=b_{1}+\left(1+b_{1}\right) z^{2}+\left(1-a_{3}^{2} b_{2}\right) z^{4}>0$.

Therefore

$$
\widehat{U}(z, t)=\exp (-t A(z)) \hat{\varphi}(z) .
$$

The eigenvalues of the matrix $A(z)$ are given by

$$
\begin{aligned}
& \lambda_{1}=\lambda_{1}(z)=\frac{z^{2}}{2 \sigma(z)}\left(\left(b_{1}+z^{2}\right) \varepsilon_{1}+\left(1+z^{2}\right) \varepsilon_{2}+\delta(z)\right) \\
& \lambda_{2}=\lambda_{2}(z)=\frac{z^{2}}{2 \sigma(z)}\left(\left(b_{1}+z^{2}\right) \varepsilon_{1}+\left(1+z^{2}\right) \varepsilon_{2}-\delta(z)\right),
\end{aligned}
$$


where

$$
\delta(z)=\left\{\left[\left(b_{1}+z^{2}\right) \varepsilon_{1}-\left(1+z^{2}\right) \varepsilon_{2}\right]^{2}+4 a_{3}^{2} b_{2} \varepsilon_{1} \varepsilon_{2} z^{4}\right\}^{1 / 2} .
$$

Computing the matrix $\exp (-t(A(z))$ and using (2.5) we find

$$
\begin{aligned}
& \hat{u}(z, t)=a_{11}(z, t) \hat{\varphi}_{1}(z)+a_{12}(z, t) \hat{\varphi}_{2}(z) \\
& \hat{v}(z, t)=a_{21}(z, t) \hat{\varphi}_{1}(z)+a_{22}(z, t) \hat{\varphi}_{2}(z)
\end{aligned}
$$

where

$$
\begin{aligned}
& a_{11}(z, t)=\left(\lambda_{1}-\lambda_{2}\right)^{-1}\left[\left(\lambda_{1}-d\right) \exp \left(-t \lambda_{1}\right)-\left(\lambda_{2}-d\right) \exp \left(-t \lambda_{2}\right)\right] \\
& a_{12}(z, t)=\left(\lambda_{1}-\lambda_{2}\right)^{-1} c^{-1}\left(\lambda_{1}-d\right)\left(\lambda_{2}-d\right)\left[\exp \left(-t \lambda_{1}\right)-\exp \left(-t \lambda_{2}\right)\right] \\
& a_{21}(z, t)=\left(\lambda_{1}-\lambda_{2}\right)^{-1} c\left[\exp \left(-t \lambda_{2}\right)-\exp \left(-t \lambda_{1}\right)\right] \\
& a_{22}(z, t)=\left(\lambda_{1}-\lambda_{2}\right)^{-1}\left[\left(\lambda_{1}-d\right) \exp \left(-t \lambda_{2}\right)-\left(\lambda_{2}-d\right) \exp \left(-t \lambda_{1}\right)\right]
\end{aligned}
$$

and

$$
c=-b_{2} a_{3} \varepsilon_{1} \frac{z^{4}}{\sigma(z)}, \quad d=\left(1+z^{2}\right) \varepsilon_{2} \frac{z^{2}}{\sigma(z)} .
$$

Using the inverse of the Fourier transform in (2.6) we obtain

$$
\begin{aligned}
& u(x, t)=\frac{1}{\sqrt{2 \pi}} \int_{-\infty}^{\infty}\left[a_{11}(z, t) \hat{\varphi}_{1}(z)+a_{12}(z, t) \hat{\varphi}_{2}(z)\right] \exp (i x z) d z \\
& v(x, t)=\frac{1}{\sqrt{2 \pi}} \int_{-\infty}^{\infty}\left[a_{21}(z, t) \hat{\varphi}_{1}(z)+a_{22}(z, t) \hat{\varphi}_{2}(z)\right] \exp (i x z) d z
\end{aligned}
$$

We also obtain from (2.7) the following inequalities

$$
\left|a_{i j}(z, t)\right| \leq \alpha \exp \left(-\frac{z^{2}}{1+z^{2}} \beta t\right), \quad i, j=1,2
$$

where

and

$$
\alpha=\max \left\{1,\left(\frac{\varepsilon_{2}}{b_{2} \varepsilon_{1}}\right)^{\frac{1}{2}}, \frac{1}{2}\left(\frac{b_{2} \varepsilon_{1}}{\varepsilon_{2}}\right)^{\frac{1}{2}}\right\}
$$

$$
\beta=\varepsilon_{1} \varepsilon_{2}\left\{\max \left[b_{1} \varepsilon_{1}+\varepsilon_{2}, \varepsilon_{1}+\varepsilon_{2}+2\left|a_{3}\right|\left(b_{2} \varepsilon_{1} \varepsilon_{2}\right)^{1 / 2}\right]\right\}^{-1} .
$$

Remark. In the above calculation we assumed that $a_{3} \neq 0$. In the case $a_{3}=0$ we have

$$
\begin{aligned}
& a_{11}(z, t)=\exp \left(-\frac{z^{2}}{1+z^{2}} \varepsilon_{1} t\right), a_{22}(z, t)=\exp \left(-\frac{z^{2}}{1+z^{2}} \varepsilon_{2} t\right), \\
& a_{12}(z, t)=a_{21}(z, t)=0,
\end{aligned}
$$


and we can replace the values of $\alpha$ and $\beta$ in (2.10) by $\alpha=1$ and $\beta=$ $\min \left(\varepsilon_{1}, \frac{\varepsilon_{2}}{\min \left(1, b_{1}\right)}\right)$ without changes on the final results stated in the next theorem.

Theorem 2.3. Let $\varphi \in H^{s} \times H^{s}$ for $s \geq 2$ and $a_{3}^{2} b_{2}<1$. Then we have the following estimate for the solution of (2.1)-(2.2):

$$
\|(u, v)\|_{L^{\infty} \times L^{\infty}} \leq C(1+t)^{-1 / 4}\|\varphi\|_{H^{1} \times H^{1}} .
$$

Moreover, if also $\varphi \in L^{1} \times L^{1}$, then

$$
\|(u, v)\|_{L^{\infty} \times L^{\infty}} \leq C(1+t)^{-1 / 2}\left(\|\varphi\|_{H^{1} \times H^{1}}+\|\varphi\|_{L^{1} \times L^{1}}\right)
$$

and

$$
\|(u, v)\|_{L^{2} \times L^{2}} \leq C(1+t)^{-1 / 4}\left(\|\varphi\|_{L^{2} \times L^{2}}+\|\varphi\|_{L^{1} \times L^{1}}\right) .
$$

Proof. Taking the norm $L^{\infty}$ in (2.8) and using (2.10) we obtain

$$
\begin{aligned}
& \|u(\cdot, t)\|_{L^{\infty}} \leq \frac{1}{\sqrt{2 \pi}} \int_{-\infty}^{\infty}\left|a_{11}(z, t)\right|\left|\hat{\varphi}_{1}(z)\right| d z \\
& +\frac{1}{\sqrt{2 \pi}} \int_{-\infty}^{\infty}\left|a_{12}(z, t)\right|\left|\hat{\varphi}_{2}(z)\right| d z \\
& \leq \frac{\alpha}{\sqrt{2 \pi}}\left[\int_{-\infty}^{\infty} \frac{1}{1+z^{2}} \exp \left(-\frac{2 z^{2}}{1+z^{2}} \beta t\right)\right]^{1 / 2}\left(\left\|\varphi_{1}\right\|_{H^{1}}+\left\|\varphi_{2}\right\|_{H^{1}}\right) .
\end{aligned}
$$

Observe that

$$
\begin{aligned}
& \int_{|z| \leq 1} \frac{1}{1+z^{2}} \exp \left(-\frac{2 z^{2}}{1+z^{2}} \beta t\right) d z \leq 2 e^{\beta} \int_{0}^{1} e^{-\beta(1+t) z^{2}} d z \\
& \leq e^{\beta} \beta^{-\frac{1}{2}} \Gamma\left(\frac{1}{2}\right)(1+t)^{-\frac{1}{2}}
\end{aligned}
$$

and

$$
\int_{|z| \geq 1} \frac{1}{1+z^{2}} \exp \left(-\frac{2 z^{2}}{1+z^{2}} \beta t\right) d z \leq 2 e^{-\beta t} \int_{0}^{\infty} \frac{d z}{1+z^{2}} \leq C(1+t)^{-1 / 2}
$$

for all $t \geq 0$, where in $(2.15) \Gamma(\cdot)$ denotes the classical Gamma function. 
Substitution of (2.15) and (2.16) in (2.14) give us the estimate

$$
\|u(\cdot, t)\|_{L^{\infty}} \leq C(1+t)^{-1 / 4}\|\varphi\|_{H^{1} \times H^{1}}, \quad t \geq 0 .
$$

Similarly, using (2.9) and (2.10) we prove that (2.17) is also true for $v(\cdot, t)$. This proves $(2.11)$.

Now, observe that

$$
\begin{aligned}
& \frac{1}{\sqrt{2 \pi}} \int_{|z| \leq 1}\left|a_{1 j}(z, t)\right|\left|\hat{\varphi}_{j}(z)\right| d z \\
& \leq \frac{\alpha}{\sqrt{2 \pi}} \int_{|z| \leq 1} \exp \left(-\frac{z^{2}}{1+z^{2}} \beta t\right)\left|\hat{\varphi}_{j}(z)\right| d z \\
& \leq \frac{\alpha}{\pi} e^{\frac{1}{2} \beta}\left(\int_{0}^{1} e^{-\frac{1}{2} \beta(1+t) z^{2}} d z\right)\left\|\varphi_{j}\right\|_{L^{1}} \\
& \leq \frac{\alpha}{\pi \sqrt{2}} e^{\frac{1}{2} \beta} \beta^{-\frac{1}{2}} \Gamma\left(\frac{1}{2}\right)(1+t)^{-\frac{1}{2}}\left\|\varphi_{j}\right\|_{L^{1}}
\end{aligned}
$$

and

$$
\begin{aligned}
& \frac{1}{\sqrt{2 \pi}} \int_{|z| \geq 1}\left|a_{1 j}(z, t)\right|\left|\hat{\varphi}_{j}(z)\right| d z \leq \frac{2 \alpha}{\sqrt{2 \pi}}\left(\int_{1}^{\infty} \frac{e^{-2 \beta t}}{1+z^{2}} d z\right)^{\frac{1}{2}}\left\|\varphi_{j}\right\|_{H^{1}} \\
& \leq C e^{-\beta t}\left\|\varphi_{j}\right\|_{H^{1}} \leq C(1+t)^{-\frac{1}{2}}\left\|\varphi_{j}\right\|_{H^{1}}, \quad j=1,2
\end{aligned}
$$

for all $t \geq 0$.

Substitution of these inequalities in (2.14) give us that

$$
\|u(\cdot, t)\|_{L^{\infty}} \leq C(1+t)^{-\frac{1}{2}}\left(\|\varphi\|_{H^{1} \times H^{1}}+\|\varphi\|_{L^{1} \times L^{1}}\right), \quad t \geq 0 .
$$

A similar estimate can be proved for $v(\cdot, t)$. This proves (2.12).

Finally, using Plancherel's theorem and (2.6) we obtain

$$
\begin{aligned}
\|u(\cdot, t)\|_{L^{2}}^{2}= & \|\hat{u}(\cdot, t)\|_{L^{2}}^{2} \leq 2 \int_{-\infty}^{\infty}\left|a_{11}(z, t)\right|^{2}\left|\hat{\varphi}_{1}(z)\right|^{2} d z \\
& +2 \int_{-\infty}^{\infty}\left|a_{12}(z, t)\right|^{2}\left|\hat{\varphi}_{2}(z)\right|^{2} d z .
\end{aligned}
$$

Estimating each integral in (2.19) as before we have

$$
\begin{aligned}
& 2 \int_{-\infty}^{\infty}\left|a_{1 j}(z, t)\right|^{2}\left|\hat{\varphi}_{j}(z)\right|^{2} d z \leq \frac{\alpha^{2}}{\pi} e^{\beta} \beta^{-\frac{1}{2}} \Gamma\left(\frac{1}{2}\right)(1+t)^{-\frac{1}{2}}\left\|\varphi_{j}\right\|_{L^{1}}^{2} \\
& +4 \alpha^{2} e^{-\beta t}\left\|\varphi_{j}\right\|_{L^{2}}^{2} \leq C(1+t)^{-\frac{1}{2}}\left(\left\|\varphi_{j}\right\|_{L^{1}}^{2}+\left\|\varphi_{j}\right\|_{L^{2}}^{2}\right), \quad t \geq 0,
\end{aligned}
$$


Thus

$$
\|u(\cdot, t)\|_{L^{2}} \leq C(1+t)^{-\frac{1}{4}}\left(\|\varphi\|_{L^{2} \times L^{2}}+\|\varphi\|_{L^{1} \times L^{1}}\right), \quad t \geq 0 .
$$

A similar estimate is also true for $v(\cdot, t)$. This proves (2.13).

Remark. Under the hypothesis of Theorem 2.3, using interpolation we immediately obtain

$$
\|(u, v)\|_{L^{q} \times L^{q}} \leq C(1+t)^{-\frac{1}{2}\left(1-\frac{1}{q}\right)}\left(\|\varphi\|_{H^{1} \times H^{1}}+\|\varphi\|_{L^{1} \times L^{1}}\right)
$$

for all $2 \leq q \leq \infty$. We also easily obtain from (2.8)-(2.9) and (2.10) the following estimate

$$
\|E(t) \varphi\|_{H^{s} \times H^{s}} \leq \sqrt{2 \alpha}\|\varphi\|_{H^{s} \times H^{s}}, \quad t>0 .
$$

\section{Existence of solutions of the nonlinear system}

In this section we shall prove that the initial value problem (1.4)-(1.5) has a unique global solution $U(\cdot, t)$ in the class $C^{1}\left([0, \infty) ; H^{s} \times H^{s}\right)$, provided that $s \geq 2, a_{3}^{2} b_{2}<1$ and $\varphi \in H^{s} \times H^{s}$ and that this solution depends continuously on the initial data.

We begin observing that for all $U, V$ in $H^{s} \times H^{s}$ the following inequalities are true

$$
\begin{aligned}
& \|F(U)-F(V)\|_{H^{s} \times H^{s}} \\
& \leq C_{1}\left(\|U\|_{H^{s} \times H^{s}}+\|V\|_{H^{s} \times H^{s}}\right)^{p}\|U-V\|_{H^{s} \times H^{s}}, \quad s \geq 1 \\
& \quad\left\|M^{-1} \partial_{x}[F(U)-F(V)]\right\|_{H^{s} \times H^{s}} \\
& \quad \leq C_{0} C_{1}\left(\|U\|_{H^{s-1} \times H^{s-1}}\right. \\
& \left.\quad+\|V\|_{H^{s-1} \times H^{s-1}}\right)^{p}\|U-V\|_{H^{s-1} \times H^{s-1}}, \quad s \geq 2
\end{aligned}
$$

where $F$ is defined in (1.7) and $C_{0}, C_{1}$ are positive constants with $C_{0}$ as in the Corollary 2.2.

The proof of (3.1) follows easily from the fact that when $s>\frac{1}{2}$, $H^{s}(\mathbb{R})$ is a Banach algebra and (3.2) is an immediate consequence of Corollary 2.2 and (3.1).

Theorem 3.1. (Local existence). Let $s \geq 2, \quad a_{3}^{2} b_{2}<1$ and $\varphi \in H^{s} \times$ $H^{s}$. Then there exists $T>0$ and a unique solution $U \in C^{1}\left([0, T] ; H^{s} \times\right.$ $\left.H^{s}\right)$ of (1.4)-(1.5). 
Proof. Using Lemma 2.1 we can write the integral equation associated with (1.4)-(1.5):

$$
U(\cdot, t)=E(t) \varphi-\int_{0}^{t} E(t-\sigma) M^{-1} \partial_{x} F(U(\cdot, \sigma)) d \sigma
$$

where $E(t)$ denotes the semigroup generated by $\mathcal{L}=M^{-1} B \frac{\partial^{2}}{\partial x^{2}}$. We consider the space of functions

$$
\begin{gathered}
Y_{T}=\left\{U \in C\left([0, T] ; H^{s} \times H^{s}\right)\right. \text { such that } \\
\left.\sup _{0 \leq t \leq T}\|U(\cdot, t)-E(t) \varphi\|_{H^{s} \times H^{s}} \leq 1 \text { and } U(\cdot, 0)=\varphi\right\}
\end{gathered}
$$

with the norm $\|U\|_{Y_{T}}=\sup _{0 \leq t \leq T}\|U(\cdot, t)\|_{H^{s} \times H^{s}}$, where $s \geq 2$.

Let us denote by $\mathcal{P}(U)(\cdot, t)$ the right-hand side of (3.3). Using inequality (3.1) we easily see that $\mathcal{P}: Y_{T} \rightarrow Y_{T}$ is a contraction if $T$ is chosen sufficiently small. (In fact it sufficient to take $T$ as $T=\left[2^{p} C_{1} \sqrt{2 \alpha}(1+\right.$ $\left.\left.\sqrt{2 \alpha}\|\varphi\|_{H^{s} \times H^{s}}\right)^{p+1}\right]^{-1}$, where $C_{1}$ and $\alpha$ are defined in (3.1) and (2.20) respectively). By Banach Fixed Point Theorem it follows that there exists a unique $U \in Y_{T}$ satisfying (3.3).

To calculate $U_{t}$ let $V(t)=\int_{0}^{t} E(t-\sigma) M^{-1} \partial_{x} F(U(\cdot, \sigma)) d \sigma, 0 \leq t \leq T$. A direct calculation shows that

$$
V_{t}(\cdot, t)=\mathcal{L}\left[\int_{0}^{t} E(t-\sigma) M^{-1} \partial_{x} F(U(\cdot, \sigma)) d \sigma\right]+M^{-1} \partial_{x} F(U(\cdot, t)) .
$$

Since $U(\cdot, t)=E(t) \varphi-V(t)$, then we conclude that

$$
\begin{aligned}
U_{t}(\cdot, t) & =\mathcal{L} U(\cdot, t)-M^{-1} \partial_{x} F(U(\cdot, t)) \\
& =M^{-1} B U_{x x}(\cdot, t)-M^{-1} \partial_{x} F(U(\cdot, t)),
\end{aligned}
$$

for all $0 \leq t \leq T$. This proves, in view of Corollary 2.2 and inequality (3.1), that $U_{t} \in C\left([0, T] ; H^{s} \times H^{s}\right)$ and that $U(\cdot, t)$ satisfies (1.4). Obviously $U(\cdot, 0)=\varphi$.

To prove uniqueness observe that if $U, V$ are any solutions of (1.4)(1.5) in the class $C^{1}\left([0, T] ; H^{s} \times H^{s}\right)$ with $s \geq 2$, then $W=U-V$ is also in the same class and satisfies

$$
W_{t}=\mathcal{L} W-M^{-1} \partial_{x}[F(U)-F(V)], \quad W(\cdot, 0)=0 .
$$


Using (3.4) we have that

$$
\frac{d}{d \sigma} E(t-\sigma) W(\cdot, \sigma)=-E(t-\sigma) M^{-1} \partial_{x}[F(U(\cdot, \sigma))-F(V(\cdot, \sigma))] .
$$

Integrating this equation from 0 to $t$ we obtain

$$
W(\cdot, t)=-\int_{0}^{t} E(t-\sigma) M^{-1} \partial_{x}[F(U(\cdot \sigma))-F(V(\cdot, \sigma))] d \sigma .
$$

This equation with (2.20) and (3.2) imply that

$$
\begin{gathered}
\|W(\cdot, t)\|_{H^{s} \times H^{s}} \\
\leq \sqrt{2 \alpha} C_{0} C_{1} \sup _{0 \leq t \leq T}\left(\|U(\cdot, t)\|_{H^{s} \times H^{s}}+\right. \\
\left.\|V(\cdot, t)\|_{H^{s} \times H^{s}}\right)^{p} \int_{0}^{t}\|W(\cdot, \sigma)\|_{H^{s} \times H^{s}} d \sigma .
\end{gathered}
$$

By Gronwall's inequality we obtain $W=0$.

Using well known techniques (see [13] for example) we obtain the following

Corollary 3.2. Under the hypothesis of Theorem 3.1 there exists a unique solution $U \in C^{1}\left(\left[0, T^{*}\right) ; H^{s} \times H^{s}\right)$ of (1.4)-(1.5) with the property that either $T^{*}=\infty$ or $T^{*}<\infty$ and $\lim _{t \uparrow T^{*}}\|U(\cdot, t)\|_{H^{s} \times H^{s}}=\infty$.

Lemma 3.3. Let $s \geq 2, a_{3}^{2} b_{2}<1$ and $\varphi=\left(\varphi_{1}, \varphi_{2}\right) \in H^{s} \times H^{s}$. If $U \in C^{1}\left([0, T) ; H^{s} \times H^{s}\right)$ is a solution of (1.4)-(1.5), then there exists a positive constant $C_{2}=C_{2}\left(b_{1}, b_{2}, a_{3}\right)$ such that

$$
\|U(\cdot, t)\|_{H^{1} \times H^{1}} \leq C_{2}\|\varphi\|_{H^{1} \times H^{1}}, \quad 0 \leq t<T .
$$

Proof. Let $u, v$ be the components of $U$. Multiply the first equation in (1.1) by $b_{2} u$ and the second equation by $v$, integrate in the whole space and add to obtain

$$
\begin{aligned}
& \frac{1}{2} \frac{d}{d t}\left\{b_{2}\|u\|_{L^{2}}^{2}+b_{2}\left\|u_{x}\right\|_{L^{2}}^{2}+b_{1}\|v\|_{L^{2}}^{2}+\left\|v_{x}\right\|_{L^{2}}^{2}+2 a_{3} b_{2}\left(u_{x}, v_{x}\right)_{L^{2}}\right\} \\
& +b_{2} \varepsilon_{1}\left\|u_{x}\right\|_{L^{2}}^{2}+\varepsilon_{2}\left\|v_{x}\right\|_{L^{2}}^{2}=0 .
\end{aligned}
$$


Integration of (3.6) in time give us

$$
\begin{aligned}
& b_{2}\|u\|_{L^{2}}^{2}+b_{2}\left\|u_{x}\right\|_{L^{2}}^{2}+b_{1}\|v\|_{L^{2}}^{2}+\left\|v_{x}\right\|_{L^{2}}^{2}+2 a_{3} b_{2}\left(u_{x}, v_{x}\right)_{L^{2}} \\
& +2 \int_{0}^{t}\left(b_{2} \varepsilon_{1}\left\|u_{x}(\cdot, \sigma)\right\|_{L^{2}}^{2}+\varepsilon_{2}\left\|v_{x}(\cdot, \sigma)\right\|_{L^{2}}^{2}\right) d \sigma=b_{2}\left\|\varphi_{1}\right\|_{L^{2}}^{2} \\
& +b_{2}\left\|\partial_{x} \varphi_{1}\right\|_{L^{2}}^{2}+b_{1}\left\|\varphi_{2}\right\|_{L^{2}}^{2}+\left\|\partial_{x} \varphi_{2}\right\|_{L^{2}}^{2}+2 a_{3} b_{2}\left(\partial_{x} \varphi_{1}, \partial_{x} \varphi_{2}\right)_{L^{2}} .
\end{aligned}
$$

Observe that if $a_{3}^{2} b_{2}<\xi<1$, then $\left|2 a_{3}^{2} b_{2}\left(u_{x}, v_{x}\right)_{L^{2}}\right| \leq b_{2} \xi\left\|u_{x}\right\|_{L^{2}}^{2}+$ $\frac{a_{3}^{2} b_{2}}{\xi}\left\|v_{x}\right\|_{L^{2}}^{2}$. Using this fact in (3.7) we obtain

$$
\begin{aligned}
& b_{2}\|u\|_{L^{2}}^{2}+b_{2}(1-\xi)\left\|u_{x}\right\|_{L^{2}}^{2}+b_{1}\|v\|_{L^{2}}^{2}+\left(1-\frac{a_{3}^{2} b_{2}}{\xi}\right)\left\|v_{x}\right\|_{L^{2}}^{2} \\
& +2 \int_{0}^{t}\left(b_{2} \varepsilon_{1}\left\|u_{x}(\cdot, \sigma)\right\|_{L^{2}}^{2}+\varepsilon_{2}\left\|v_{x}(\cdot, \sigma)\right\|_{L^{2}}^{2}\right) d \sigma \\
& \leq b_{2}\left\|\varphi_{1}\right\|_{L^{2}}^{2}+b_{2}(1+\xi)\left\|\partial_{x} \varphi_{1}\right\|_{L^{2}}^{2}+b_{1}\left\|\varphi_{2}\right\|_{L^{2}}^{2}+\left(1+\frac{a_{3}^{2} b_{2}}{\xi}\right)\left\|\partial_{x} \varphi_{2}\right\|_{L^{2}}^{2} .
\end{aligned}
$$

Now, choosing $\xi=\frac{1}{2}\left(a_{3}^{2} b_{2}+1\right)$ in (3.8) we obtain (3.5), with $C_{2}$ depending only on $b_{1}, b_{2}$ and $a_{3}$.

Theorem 3.4. (Global existence). Let $s \geq 2, a_{3}^{2} b_{2}<1$ and $\varphi \in H^{s} \times$ $H^{s}$. Then the initial value problem (1.4)-(1.5) has a unique solution $U \in C^{1}\left([0, \infty) ; H^{s} \times H^{s}\right)$.

Proof. By Theorem 3.1 and Corollary 3.2 it sufficient to prove that if $U$ is any solution of (1.4)-(1.5) in the class $C^{1}\left([0, T) ; H^{s} \times H^{s}\right), s \geq 2$, then there exists a positive constant $C=C\left(s, T,\|\varphi\|_{H^{s} \times H^{s}}\right)$ such that $\sup _{0<t<T}\|U(\cdot, t)\|_{H^{s} \times H^{s}} \leq C$. Since $U$ also satisfies the integral equation (3.3) for $0 \leq t<T$, then using (2.20) and (3.2) we have that

$$
\begin{gathered}
\|U(\cdot, t)\|_{H^{s} \times H^{s}} \\
\leq \sqrt{2 \alpha}\|\varphi\|_{H^{s} \times H^{s}}+\sqrt{2 \alpha} C_{0} C_{1} \int_{0}^{t}\|U(\cdot, \sigma)\|_{H^{s-1} \times H^{s-1}}^{p+1} d \sigma .
\end{gathered}
$$

From (3.9) and (3.5) it follows that

$\|U(\cdot, t)\|_{H^{2} \times H^{2}} \leq \sqrt{2 \alpha} \max \left(1, C_{2}^{p+1}\right)\left(1+C_{0} C_{1} T\|\varphi\|_{H^{2} \times H^{2}}^{p}\right)\|\varphi\|_{H^{2} \times H^{2}}$. 
By induction we obtain

$$
\begin{gathered}
\sup _{0 \leq t<T}\|U(\cdot, t)\|_{H^{m} \times H^{m}} \\
\leq \eta^{(p+2)^{m-2}}\left(1+C_{0} C_{1} T\|\varphi\|_{H^{m} \times H^{m}}\right)^{(p+2)^{m-2}}\|\varphi\|_{H^{m} \times H^{m}}
\end{gathered}
$$

where $m=2,3, \ldots$ and $\eta=\sqrt{2 \alpha} \max \left(1, C_{2}^{p+1}\right)$.

Now, for any $s>2$ we choose an integer $m$ such that $m<s \leq m+1$ and use (3.9), (3.10) and the Sobolev imbeddings $H^{s} \hookrightarrow H^{m} \hookrightarrow H^{s-1}$ to conclude that

$$
\begin{aligned}
\|U(\cdot, t)\|_{H^{*} \times H^{*}} & \leq \sqrt{2 \alpha}\|\varphi\|_{H^{*} \times H^{*}}+\sqrt{2 \alpha} C_{0} C_{1} \int_{0}^{t}\|U(\cdot, \sigma)\|_{H^{m} \times H^{m}}^{p+1} d \sigma \\
& \leq \eta^{(p+2)^{m-1}}\left(1+C_{0} C_{1} T\|\varphi\|_{H^{*} \times H^{*}}^{p}\right)^{(p+2)^{m-1}}\|\varphi\|_{H^{*} \times H^{*}} \\
& \leq \eta^{(p+2)^{s-1}}\left(1+C_{0} C_{1} T\|\varphi\|_{H^{*} \times H^{*}}^{p}\right)^{(p+2)^{s-1}}\|\varphi\|_{H^{*} \times H^{*}}
\end{aligned}
$$

for all $0 \leq t<T$. This proves the theorem.

The solution of (1.4)-(1.5) obtained in Theorem 3.4 depends continuously on the initial data in the following sense:

Theorem 3.5. (Continuous dependence). Let $s \geq 2, a_{3}^{2} b_{2}<1$ and $U_{n}, U$ the solutions of (1.4)-(1.5) in $C^{1}\left([0, \infty) ; H^{s} \times H^{s}\right)$ corresponding to the initial data $\varphi_{n}, \varphi$ in $H^{s} \times H^{s}$, respectively. If $\varphi_{n} \rightarrow \varphi$ in $H^{s} \times H^{s}$ then

$$
\begin{aligned}
& \lim _{n \rightarrow \infty} \sup _{0 \leq t \leq T}\left(\left\|U_{n}(\cdot, t)-U(\cdot, t)\right\|_{H^{s} \times H^{s}}\right. \\
& \left.+\left\|\partial_{t} U_{n}(\cdot, t)-\partial_{t} U(\cdot, t)\right\|_{H^{s} \times H^{s}}\right)=0,
\end{aligned}
$$

for all fixed $T>0$.

Proof. Fixed $T>0$, using the integral equation (3.3) with $\varphi_{n}, \varphi$ and $(2.20),(3.2)$ we obtain the estimate

$$
\begin{gathered}
\left\|U_{n}(\cdot, t)-U(\cdot, t)\right\|_{H^{s} \times H^{s}} \leq \sqrt{2 \alpha}\left\|\varphi_{n}-\varphi\right\|_{H^{s} \times H^{s}}+ \\
\sqrt{2 \alpha} C_{0} C_{1} \sup _{0 \leq t \leq T}\left(\left\|U_{n}(\cdot, t)\right\|_{H^{s} \times H^{s}}+\right. \\
\left.\|U(\cdot, t)\|_{H^{s} \times H^{s}}\right)^{p} \int_{0}^{t}\left\|U_{n}(\cdot, \sigma)-U(\cdot, \sigma)\right\|_{H^{s} \times H^{s}} d \sigma
\end{gathered}
$$


for all $0 \leq t \leq T$. Therefore, Gronwall's inequality implies that

$$
\sup _{0 \leq t \leq T}\left\|U_{n}(\cdot, t)-U(\cdot, t)\right\|_{H^{s} \times H^{s}} \leq \sqrt{2 \alpha}\left\|\varphi_{n}-\varphi\right\|_{H^{s} \times H^{s}} e^{\omega_{n} T}
$$

where

$\omega_{n}=\sqrt{2 \alpha} C_{0} C_{1} \sup _{0 \leq t \leq T}\left(\left\|U_{n}(\cdot, t)\right\|_{H^{s} \times H^{s}}+\|U(\cdot, t)\|_{H^{s} \times H^{s}}\right)^{p}, n=1,2, \ldots$

Moreover, using (1.4) with $U_{n}$ and $U$ and (3.11) we have

$$
\begin{aligned}
& \quad\left\|\partial_{t} U_{n}(\cdot, t)-\partial_{t} U(\cdot, t)\right\|_{H^{s} \times H^{s}} \\
& \leq\left\|\mathcal{L} U_{n}(\cdot, t)-\mathcal{L} U(\cdot, t)\right\|_{H^{s} \times H^{s}} \\
& +\| M^{-1} \partial_{x}\left[F\left(U_{n}(\cdot, t)-F(U(\cdot, t))\right] \|_{H^{s} \times H^{s}}\right. \\
& \leq C_{0} \max \left(\varepsilon_{1} b_{1}, b_{2}\right)\left\|U_{n}(\cdot, t)-U(\cdot, t)\right\|_{H^{s} \times H^{s}} \\
& +\omega_{n}\left\|U_{n}(\cdot, t)-U(\cdot, t)\right\|_{H^{s} \times H^{s}} \\
& \leq \sqrt{2 \alpha}\left[C_{0} \max \left(\varepsilon_{1} b_{1}, b_{2}\right)+\omega_{n}\right] e^{\omega_{n} T}\left\|\varphi_{n}-\varphi\right\|_{H^{s} \times H^{s}}, \quad 0 \leq t \leq T .
\end{aligned}
$$

Since $\varphi_{n} \rightarrow \varphi$ in $H^{s} \times H^{s}$, then using the same arguments presented in the proof of Theorem 3.4 we show that $\left(\omega_{n}\right)$ is a bounded sequence. Thus, the conclusion of Theorem 3.5 follows from (3.11) and (3.12).

Remarks. (1) The same results given by Theorems $3.1,3.4$ and 3.5 above can be proved for (1.3) in the case of $\varepsilon_{1}=\varepsilon_{2}=0$. In fact, for this case we can replace the hypothesis $s \geq 2$ by $s \geq 1$. These results improve those one presented in [5].

(2) It is also important to observe that in some particular cases, for example, $b_{1}=b_{2}=1, a_{1}=a_{2},\left|a_{3}\right|<1$ and $\varepsilon_{1}=\varepsilon_{2}=0$, system (1.1) has solitary wave solutions of the form $u(x, t)=v(x, t)=\psi(x-c t)$, where $c>0$ and

$$
\psi(x)=\left[\frac{1}{2}(p+1)(p+2) \frac{c}{1+a_{1}(p+1)} \operatorname{sech}^{2} \frac{p}{2}\left(\frac{1}{1+a_{3}}\right)^{1 / 2} x\right]^{1 / p}
$$

Thus, an argument similar to that of page 579 of [9] shows that (in this case) the continuous dependence in Theorem 3.5 can't be uniform for all $t>0$. 


\section{Decay estimates to the nonlinear system}

Let us denote by $u, v$ the components of the global solution $U$ of (1.4)(1.5) obtained in the last section. From Theorem 3.4 and Lemma 3.3 we deduce that

$$
\int_{0}^{\infty}\left(\left\|u_{x}(\cdot, \sigma)\right\|_{L^{2}}^{2}+\left\|v_{x}(\cdot, \sigma)\right\|_{L^{2}}^{2}\right) d \sigma<+\infty .
$$

Theorem 4.1. Under the conditions of Theorem 3.4 and $p \geq 3$ we have the following decay estimate

$$
U(\cdot, t)\left\|_{L^{\infty} \times L^{\infty}} \leq C(1+t)^{-\frac{1}{4}}\right\| \varphi \|_{H^{1} \times H^{1}}, \quad t \geq 0 .
$$

Moreover, if also $\varphi \in L^{1} \times L^{1}$, then

$$
\|U(\cdot, t)\|_{L^{q} \times L^{q}} \leq C(1+t)^{-\frac{1}{2}\left(1-\frac{1}{q}\right)}\left(\|\varphi\|_{H^{1} \times H^{1}}+\|\varphi\|_{L^{1} \times L^{1}}\right), \quad t \geq 0
$$

for all $2 \leq q \leq \infty$.

Proof. Since $U(\cdot, t)$ also satisfies the integral equation (3.3) for any $t \geq 0$, then we can take the norm $L^{\infty} \times L^{\infty}$ in (3.3) and use (2.11) to obtain

$$
\begin{aligned}
& \|U(\cdot, t)\|_{L^{\infty} \times L^{\infty}} \leq C(1+t)^{-\frac{1}{4}}\|\varphi\|_{H^{1} \times H^{1}} \\
& \quad+C \int_{0}^{t}(1+t-\sigma)^{-\frac{1}{4}}\left\|M^{-1} \partial_{x} F(U(\cdot, \sigma))\right\|_{H^{1} \times H^{1}} d \sigma .
\end{aligned}
$$

To estimate $\left\|M^{-1} \partial_{x} F(U)\right\|_{H^{1} \times H^{1}}$, let $\partial_{x} F(U)=g=\left(g_{1}, g_{2}\right)$, where

$$
\begin{aligned}
& g_{1}=b_{1} u^{p} u_{x}+b_{1} a_{1} v^{p} v_{x}+b_{1} a_{2}\left(u^{p} v\right)_{x} \\
& g_{2}=v^{p} v_{x}+b_{2} a_{2} u^{p} u_{x}+b_{2} a_{1}\left(u v^{p}\right)_{x} .
\end{aligned}
$$

From Corollary 2.2 it follows that

$$
\left\|M^{-1} \partial_{x} F(U)\right\|_{H^{1} \times H^{1}} \leq C_{o}\|g\|_{L^{2} \times L^{2}}
$$

Using the inequality $\|w\|_{L^{\infty}} \leq\|w\|_{L^{2}}^{1 / 2}\|w\|_{L^{2}}^{1 / 2}$ valid for any $w \in H^{1}(\mathbb{R})$, the imbedding $H^{1} \hookrightarrow L^{\infty}$, and lemma 3.3 we estimate $\left\|g_{1}\right\|_{L^{2}}$ as follows

$$
\left\|g_{1}\right\|_{L^{2}} \leq b_{1}\|u\|_{L^{\infty}}^{p-2}\left\|u_{x}\right\|_{L^{2}}^{2}\|u\|_{L^{2}}+b_{1}\left|a_{1}\right|\|v\|_{L^{\infty}}^{p-2}\|v\|_{L^{2}}\left\|v_{x}\right\|_{L^{2}}^{2}
$$




$$
\begin{aligned}
& +b_{1} p\left|a_{2}\right|\|u\|_{L^{\infty}}^{p-3}\left\|u_{x}\right\|_{L^{2}}^{2}\|u\|_{L^{2}}\|v\|_{L^{\infty}} \\
& +b_{1}\left|a_{2}\right|\|u\|_{L^{\infty}}^{p-2}\left\|u_{x}\right\|_{L^{2}}\|u\|_{L^{2}}\left\|v_{x}\right\|_{L^{2}} \\
& \leq C\left(\left\|u_{x}\right\|_{L^{2}}^{2}+\left\|v_{x}\right\|_{L^{2}}^{2}\right)\|U\|_{L^{\infty} \times L^{\infty}}
\end{aligned}
$$

A similar estimate is also true for $\left\|g_{2}\right\|_{L^{2}}$. From these estimates and (4.5) we conclude that

$$
\left\|M^{-1} \partial_{x} F(U)\right\|_{H^{1} \times H^{1}} \leq C\left(\left\|u_{x}\right\|_{L^{2}}^{2}+\left\|v_{x}\right\|_{L^{2}}^{2}\right)\|U\|_{L^{\infty} \times L^{\infty}} .
$$

Substitution of (4.7) in (4.4) give us

$$
\begin{aligned}
\|U(\cdot t)\|_{L^{\infty} \times L^{\infty}} & \leq C(1+t)^{-\frac{1}{4}}\|\varphi\|_{H^{1} \times H^{1}} \\
& +C \int_{0}^{t}(1+t-\sigma)^{-\frac{1}{4}} H(\sigma)\|U(\cdot, \sigma)\|_{L^{\infty} \times L^{\infty}} d \sigma,
\end{aligned}
$$

where $H(t)=\left\|u_{x}(\cdot, t)\right\|_{L^{2}}^{2}+\left\|v_{x}(\cdot, t)\right\|_{L^{2}}^{2}$ is an integrable function on $[0, \infty)$ by $(4.1)$.

By a Gronwall's lemma (see Lemma 1 of [11]) it follows (4.2).

Now taking the norms $L^{\infty} \times L^{\infty}$ and $L^{2} \times L^{2}$ in (3.3) and using (2.12)-(2.13) we obtain respectively

$$
\begin{aligned}
& \|U(\cdot, t)\|_{L^{\infty} \times L^{\infty}} \leq C(1+t)^{-\frac{1}{2}}\left(\|\varphi\|_{H^{1} \times H^{1}}+\|\varphi\|_{L^{1} \times L^{1}}\right) \\
& +C \int_{0}^{t}(1+t-\sigma)^{-\frac{1}{2}}\left(\left\|M^{-1} \partial_{x} F(U(\cdot, \sigma))\right\|_{H^{1} \times H^{1}}\right. \\
& \left.+\left\|M^{-1} \partial_{x} F(U(\cdot, \sigma))\right\|_{L^{1} \times L^{1}}\right) d \sigma
\end{aligned}
$$

and

$$
\begin{aligned}
& \|U(\cdot, t)\|_{L^{2} \times L^{2}} \leq C(1+t)^{-\frac{1}{4}}\left(\|\varphi\|_{L^{2} \times L^{2}}+\|\varphi\|_{L^{1} \times L^{1}}\right) \\
& +C \int_{0}^{t}(1+t-\sigma)^{-\frac{1}{4}}\left(\left\|M^{-1} \partial_{x} F(U(\cdot, \sigma))\right\|_{L^{2} \times L^{2}}\right. \\
& \left.+\left\|M^{-1} \partial_{x} F(U(\cdot, \sigma))\right\|_{L^{1} \times L^{1}}\right) d \sigma
\end{aligned}
$$

Let us work with (4.9) first. Using the same notation as before and lemma 2.1 we have

$$
\begin{aligned}
\left\|M^{-1} \partial_{x} F(U)\right\|_{L^{2} \times L^{2}} & \leq \sum_{j=1}^{2}\left(\left\|k_{j 1} * g_{1}\right\|_{L^{2}}+\left\|k_{j 2} * g_{2}\right\|_{L^{2}}\right) \\
& \leq \sum_{j=1}^{2}\left(\left\|k_{j 1}\right\|_{L^{1}}\left\|g_{1}\right\|_{L^{2}}+\left\|k_{j 2}\right\|_{L^{1}}\left\|g_{2}\right\|_{L^{2}}\right)
\end{aligned}
$$


and

$$
\begin{aligned}
\left\|M^{-1} \partial_{x} F(U)\right\|_{L^{1} \times L^{1}} & \leq \sum_{j=1}^{2}\left(\left\|k_{j 1} * g_{1}\right\|_{L^{1}}+\left\|k_{j 2} * g_{2}\right\|_{L^{1}}\right) \\
& \leq \sum_{j=1}^{2}\left(\left\|k_{j 1}\right\|_{L^{1}}\left\|g_{1}\right\|_{L^{1}}+\left\|k_{j 2}\right\|_{L^{1}}\left\|g_{2}\right\|_{L^{1}}\right)
\end{aligned}
$$

Estimating $\left\|g_{1}\right\|_{L^{2}}$ as in (4.6) we find

$$
\left\|g_{1}\right\|_{L^{2}} \leq C\left(\left\|u_{x}\right\|_{L^{2}}^{2}+\left\|v_{x}\right\|_{L^{2}}^{2}\right)\|U\|_{L^{2} \times L^{2}} .
$$

We estimate $\left\|g_{1}\right\|_{L^{1}}$ as follows

$$
\begin{aligned}
\left\|g_{1}\right\|_{L^{1}} & \leq b_{1}\|u\|_{L^{\infty}}^{p-3}\left\|u_{x}\right\|_{L^{2}}^{2}\|u\|_{L^{2}}^{2} \\
& +b_{1}\left|a_{1}\right|\|v\|_{L^{\infty}}^{p-3}\left\|v_{x}\right\|_{L^{2}}\left\|u_{x}\right\|_{L^{2}}\|u\|_{L^{2}}\|v\|_{L^{2}} \\
& +b_{1} p\left|a_{2}\right|\|u\|_{L^{\infty}}^{p-3}\left\|u_{x}\right\|_{L^{2}}\left\|v_{x}\right\|_{L^{2}}\|u\|_{L^{2}}\|v\|_{L^{2}} \\
& +b_{1}\left|a_{2}\right|\|u\|_{L^{\infty}}^{p-3}\left\|u_{x}\right\|_{L^{2}}\left\|v_{x}\right\|_{L^{2}}\|u\|_{L^{2}}^{2} \\
& \leq C\left(\left\|u_{x}\right\|_{L^{2}}^{2}+\left\|v_{x}\right\|_{L^{2}}^{2}\right)\|U\|_{L^{2} \times L^{2}} .
\end{aligned}
$$

Similar estimates are also true for $\left\|g_{2}\right\|_{L^{2}}$ and $\left\|g_{2}\right\|_{L^{1}}$.

Thus

$$
\begin{aligned}
& \left\|M^{-1} \partial_{x} F(U)\right\|_{L^{2} \times L^{2}} \leq C\left(\left\|u_{x}\right\|_{L^{2}}^{2}+\left\|v_{x}\right\|_{L^{2}}^{2}\right)\|U\|_{L^{2} \times L^{2}} \\
& \left\|M^{-1} \partial_{x} F(U)\right\|_{L^{1} \times L^{1}} \leq C\left(\left\|u_{x}\right\|_{L^{2}}^{2}+\left\|v_{x}\right\|_{L^{2}}^{2}\right)\|U\|_{L^{2} \times L^{2}}
\end{aligned}
$$

Combining (4.11) with (4.9) we obtain

$$
\begin{array}{r}
\|U(\cdot, t)\|_{L^{2} \times L^{2}} \leq C(1+t)^{-\frac{1}{4}}\left(\|\varphi\|_{L^{2} \times L^{2}}+\|\varphi\|_{L^{1} \times L^{1}}\right)+ \\
C \int_{0}^{t}(1+t-\sigma)^{-\frac{1}{4}} H(\sigma)\|U(\cdot, \sigma)\|_{L^{2} \times L^{2}} d \sigma
\end{array}
$$

with $H(t)$ as before. Applying lemma 1 of [11] we conclude that

$$
\|U\|_{L^{2} \times L^{2}} \leq C(1+t)^{-\frac{1}{4}}\left(\|\varphi\|_{L^{2} \times L^{2}}+\|\varphi\|_{L^{1} \times L^{1}}\right), \quad t \geq 0
$$


Now, let us estimate $\left\|M^{-1} \partial_{x} F(U)\right\|_{L^{1} \times L^{1}}$ in terms of $\|U\|_{L^{\infty} \times L^{\infty}}$. Using similar arguments as before, the Young inequality and (4.12) we have

$$
\begin{gathered}
\left\|u^{p} u_{x}\right\|_{L^{1}} \leq\|u\|_{L^{\infty}}^{p-1}\|u\|_{L^{2}}\left\|u_{x}\right\|_{L^{2}} \leq\|u\|_{L^{2}}^{p / 2}\left\|u_{x}\right\|_{L^{2}}^{3 / 2}\|u\|_{L^{\infty}} \\
\leq\left(\frac{1}{4}\|u\|_{L^{2}}^{2 p}+\frac{3}{4}\left\|u_{x}\right\|_{L^{2}}^{2}\right)\|u\|_{L^{\infty}} \leq C\left((1+t)^{-\frac{p}{2}}+\left\|u_{x}\right\|_{L^{2}}^{2}\right)\|u\|_{L^{\infty}} \\
\left\|u^{p-1} u_{x} v\right\|_{L^{1}} \leq C\left((1+t)^{-\frac{p}{2}}+\left\|u_{x}\right\|_{L^{2}}^{2}\right)\|u\|_{L^{\infty}} \\
\left\|v^{p} v_{x}\right\|_{L^{1}} \leq C\left((1+t)^{-\frac{p}{2}}+\left\|v_{x}\right\|_{L^{2}}^{2}\right)\|v\|_{L^{\infty}}
\end{gathered}
$$

We also have

$$
\begin{aligned}
& \left\|u^{p} v_{x}\right\|_{L^{1}} \leq\|u\|_{L^{\infty}}^{p-2}\|u\|_{L^{2}}\left\|v_{x}\right\|_{L^{2}}\|u\|_{L^{\infty}} \\
& \leq\|u\|_{L^{2}}^{p / 2}\left\|u_{x}\right\|_{L^{2}}^{1 / 2}\left\|v_{x}\right\|_{L^{2}}\|u\|_{L^{\infty}} \\
& \leq\left(\|u\|_{L^{2}}^{2 p}+\left\|u_{x}\right\|_{L^{2}}^{2}+2\left\|v_{x}\right\|_{L^{2}}^{2}\right)\|u\|_{L^{\infty}} \\
& \leq C\left((1+t)^{-\frac{p}{2}}+\left\|u_{x}\right\|_{L^{2}}^{2}+\left\|v_{x}\right\|_{L^{2}}^{2}\right)\|u\|_{L^{\infty}}
\end{aligned}
$$

Consequently

$$
\left\|g_{1}\right\|_{L^{1}} \leq C\left((1+t)^{-\frac{p}{2}}+\left\|u_{x}\right\|_{L^{2}}^{2}+\left\|v_{x}\right\|_{L^{2}}^{2}\right)\|U\|_{L^{\infty} \times L^{\infty}}
$$

A similar estimate is also true for $\left\|g_{2}\right\|_{L^{1}}$.This fact with (4.13) and (4.10) imply that

$$
\left\|M^{-1} \partial_{x} F(U)\right\|_{L^{1} \times L^{1}} \leq C\left((1+t)^{-\frac{p}{2}}+\left\|u_{x}\right\|_{L^{2}}^{2}+\left\|v_{x}\right\|_{L^{2}}^{2}\right) \|\left. U\right|_{L^{\infty} \times L^{\infty}}
$$

Combining (4.14)-(4.7) with (4.8) we obtain

$$
\begin{aligned}
\|U(\cdot, t)\|_{L^{\infty} \times L^{\infty}} & \leq C(1+t)^{-\frac{1}{2}}\left(\|\varphi\|_{H^{1} \times H^{1}}+\|\varphi\|_{L^{1} \times L^{1}}\right) \\
& +C \int_{0}^{t}(1+t-\sigma)^{-\frac{1}{2}} \tilde{H}(\sigma)\|U(\cdot, \sigma)\|_{L^{2} \times L^{2}} d \sigma
\end{aligned}
$$

where

$$
\widetilde{H}(t)=(1+t)^{-\frac{p}{2}}+\left\|u_{x}(\cdot, t)\right\|_{L^{2}}^{2}+\left\|v_{x}(\cdot, t)\right\|_{L^{2}}^{2}
$$

is an integrable functions on $[0, \infty)$ by $(4.1)$ and because $p>2$. By lemma 1 of [11] it follows that

$$
\|U(\cdot, t)\|_{L^{\infty} \times L^{\infty}} \leq C(1+t)^{-\frac{1}{2}}\left(\|\varphi\|_{H^{1} \times H^{1}}+\|\varphi\|_{L^{1} \times L^{1}}\right), \quad t \geq 0
$$


Finally, from (4.12)-(4.15) and interpolation we obtain (4.3). This completes the proof of Theorem 4.1 .

Acknowledgements. The author would like to thank Prof. G. Perla Menzala and Prof. V. Bisognin for stimulating conversations on the subject of this paper and the referee for suggesting some improvements in the presentation and the quality of results.

\section{References}

[1] C.J. AMICK, J.L. BONA, M.E. SCHONBEK, Decay of solutions of some nonlinear wave equations, J. Diff. Equations 81 (1989), 1-49.

[2] P. BILER, Asymptotic behaviour in time of solutions to some equations generalizing the Korteweg-de Vries - Burgers equation, Bulletin Polish Acad. Sciences, Mathematics 32 (1984), 275-282.

[3] E. BISOGNIN, V. BISOGNIN, G. PERLA MENZALA, On the asymptotic behaviour in time of the solutions of a coupled system of KdV equations, Funkcialaj Ekvacioj 40 (1997), 353-370.

[4] E. BISOGNIN, V. BISOGNIN, G. PERLA MENZALA, Uniform stabilization of space-periodic solutions of a nonlinear dispersive system (submitted).

[5] V. BISOGNIN, On the asymptotic behaviour of the solutions of a nonlinear dispersive system of Benjamin-Bona-Mahony's type, Boll. Un. Mat. Ital. B (7) (1996), 99-128.

[6] V. BISOGNIN, G. PERLA MENZALA, Decay rates of the solutions of nonlinear dispersive equations, Proc. of the Royal Soc. of Edinburgh 124 A (1994), 1231-1246.

[7] J.L. BONA, L. LUO, More results on the decay of solutions to nonlinear, dispersive wave equations, Discrete and Continuous Dynamical Systems 1, № 2 (1995), 151-193.

[8] J.L. BONA, G. PONCE, J.C. SAUT, M.M. TOM, A model system for strong interaction between internal solitary waves, Comm. Math. Phys. 143 (1992), 287-313.

[9] J.L.BONA, R. SMITH, The initial-value problem for the Korteweg-de Vries equation, Philos. Trans. Royal Soc. London A, 278 (1975), 555-601.

[10] J.A. GEAR, R. GRIMSHAW, Weak and strong interactions between internal solitary waves, Studies in Appl. Math. 70 (1984), 235-258. 
[11] LINGHAI ZHANG, Decay estimates for the solutions of some nonlinear evolution equation, J. Diff. Equations 116 (1995), 31-58.

[12] J. MARSHALL ASH, J. COHEN, G. WANG, On strongly interacting internal solitary waves, The Journal of Fourier Analysis and Appl. 2, № 5 (1996), 507-517.

[13] A. PAZY, Semigroup of Linear Operators and Applications to Partial Differential Equations, Springer-Verlag, New York/Berlin, 1983.

Universidade Federal de Santa Catarina

Departamento de Matemática

88.040-900 Florianópolis

SC, Brasil

Recibido: 15 de Marzo de 1999

Revisado: 6 de Marzo de 2000 